PROCEEDINGS OF THE AMERICAN MATHEMATICAL SOCIETY

Volume 124, Number 8, August 1996

\title{
ASYMPTOTIC BEHAVIOUR OF GROUND STATES
}

\author{
JOSEPHUS HULSHOF AND ROBERTUS C. A. M. VAN DER VORST
}

(Communicated by Jeffrey Rauch)

Abstract. We derive the asymptotic behaviour of the ground states of a system of two coupled semilinear Poisson equations with a strongly indefinite variational structure in the critical Sobolev growth case.

\section{INTRODUCTION}

In this note we derive the asymptotic behaviour of the ground states of the following system of two coupled equations:

$$
-\Delta v=u^{p} ; \quad-\Delta u=v^{q} .
$$

Here $u=u(x), v=v(x), x \in \mathbf{R}^{n}, \Delta$ is the Laplace operator, $u^{p}=|u|^{p-1} u$, $v^{q}=|v|^{q-1} v$ and $p$ and $q$ are positive numbers satisfying

$$
\frac{n}{p+1}+\frac{n}{q+1}=n-2, \quad n>2 .
$$

The corresponding curve in the first quadrant of the $p, q$-plane is called the critical hyperbola. Its asymptotes are $p=\frac{2}{n-2}$ and $q=\frac{2}{n-2}$.

Definition 1. A pair $(u, v) \neq(0,0)$ is called a ground state of (1.1) if:

(a) $(u, v) \in \mathcal{D}^{2, \frac{q+1}{q}} \times \mathcal{D}^{2, \frac{p+1}{p}}$, with $p, q$ satisfying (1.2),

(b) $(u, v)$ attains the smallest nonzero critical value of

$$
J_{p, q}(u, v)=\int \nabla u \nabla v-\frac{1}{p+1} \int|u|^{p+1}-\frac{1}{q+1} \int|v|^{q+1} .
$$

Here the spaces $\mathcal{D}^{2, \frac{q+1}{q}}=\mathcal{D}^{2, \frac{q+1}{q}}\left(\mathbf{R}^{n}\right)$ and $\mathcal{D}^{2, \frac{p+1}{p}}=\mathcal{D}^{2, \frac{p+1}{p}}\left(\mathbf{R}^{n}\right)$ are defined as the completion of $C_{0}^{\infty}\left(\mathbf{R}^{n}\right)$ with respect to the norms $\|\Delta \cdot\|_{\frac{q+1}{q}}$ and $\|\Delta \cdot\|_{\frac{p+1}{p}}$ respectively.

We mention two explicit examples. Setting $u=v$ and $p=q$ reduces (1.1) to the scalar semilinear Poisson equation in the critical Sobolev case:

$$
-\Delta u=u^{p} ; \quad p=\frac{n+2}{n-2},
$$

Received by the editors February 16, 1995.

1991 Mathematics Subject Classification. Primary 35J55; Secondary 34C37.

Key words and phrases. Systems, strongly indefinite variational structure, critical Sobolev growth, ground states, asymptotic behaviour, transformation to 3- and 4-dimensional quadratic systems, heteroclinic orbits, critical point analysis.

We gratefully acknowledge the support by N.W.O., the Dutch Organisation for Scientific Research, and Enzo Mitidieri for his encouragement.

(C)1996 American Mathematical Society 
for which the ground states are given by the following family:

$$
u_{\epsilon, x_{0}}(x)=\frac{\left(n(n-2) \epsilon^{2}\right)^{\frac{n-2}{4}}}{\left(\epsilon^{2}+r^{2}\right)^{\frac{n-2}{2}}}, \quad r=\left|x-x_{0}\right| .
$$

On the other hand, if $q=1$ and $n>4$, system (1.1) can be written as the semilinear biharmonic equation with critical growth rate,

$$
\Delta^{2} u=u^{p} ; \quad p=\frac{n+4}{n-4},
$$

which has

$$
u_{\epsilon, x_{0}}(x)=\frac{\left((n-4)(n-2) n(n+2) \epsilon^{2}\right)^{\frac{n-4}{8}}}{\left(\epsilon^{2}+r^{2}\right)^{\frac{n-4}{2}}}, \quad r=\left|x-x_{0}\right|,
$$

as ground states.

Clearly these two special cases have different asymptotic behaviour. P.L. Lions [11] proved that the corresponding scalar equation for the $u$-component, which reads

$$
-\Delta\left((-\Delta u)^{1 / q}\right)=u^{p}
$$

indeed has (radial) ground states for all values of $p$ and $q$ on the critical hyperbola. However, the asymptotic behaviour of these ground states has been left open for debate [12]. Lions' result may be stated as the following existence theorem for ground states of (1.1).

Theorem 1. If $p$ and $q$ satisfy (1.2), then system (1.1) has a ground state, which is unique up to scalings and translations. Moreover the ground state is positive, radially symmetric and decreasing in $r$. The critical value of $J_{p, q}$ is given by

$$
I_{p, q}=\frac{2}{n} K_{p, q}^{\frac{n}{2}}
$$

where

$$
K_{p, q}=\min \left\{\|\Delta u\|_{\frac{q+1}{q}} ; u \in \mathcal{D}^{2, \frac{q+1}{q}},\|u\|_{p+1}=1\right\}
$$

Actually the uniqueness result is new and proved in Section 2 .

We normalize the ground state by taking it radially symmetric with respect to the origin and by setting $u(0)=1$. It follows from Theorem 1 that all the ground states of (1.1) are then given by

$$
u_{\epsilon, x_{0}}(x)=\epsilon^{-\frac{n}{p+1}} u\left(\frac{x-x_{0}}{\epsilon}\right), v_{\epsilon, x_{0}}(x)=\epsilon^{-\frac{n}{q+1}} v\left(\frac{x-x_{0}}{\epsilon}\right), \epsilon>0 .
$$

From now on we will study the normalized ground state.

Theorem 2. Assuming without restriction and throughout this note that

$$
p \leq \frac{n+2}{n-2},
$$

there exist constants $a>0$ and $b>0$ depending on $p$ and $n$, such that the normalized ground state satisfies

$$
\lim _{r \rightarrow \infty} r^{n-2} u(r)=a,
$$


while

$$
\begin{aligned}
& \lim _{r \rightarrow \infty} r^{n-2} v(r)=b \quad \text { if } p>\frac{n}{n-2}, \\
& \lim _{r \rightarrow \infty} \frac{r^{n-2}}{\log r} v(r)=b \quad \text { if } p=\frac{n}{n-2},
\end{aligned}
$$

and

$$
\lim _{r \rightarrow \infty} r^{p(n-2)-2} v(r)=b \quad \text { if } p<\frac{n}{n-2} .
$$

In the last case the constants $a$ and be are related by

$$
a^{p}=b\left(p\left(n^{2}-4\right)-2 n-p^{2}(n-2)^{2}\right) .
$$

The extension to $p>\frac{n+2}{n-2}$ follows from interchanging the role of $u$ and $v$ and of $p$ and $q$.

The exponent $\frac{n}{n-2}$ is sometimes called the Serrin exponent.

The proof of this theorem relies on the analysis of a related 3-dimensional first order system of differential equations of quadratic type. The global analysis of this quadratic system is given in Section 3. The precise asymptotics of $u$ and $v$ follow from a local critical point analysis in Section 4.

Ground states play an important role in the study of the homogeneous Dirichlet problem for perturbations of (1.1) with lower order linear terms. It is well known for instance that in the scalar case these terms (are needed for and) lead to nontrivial positive solutions [1]. Our results are needed for a generalization of these results and techniques to handle perturbations of (1.1). This will be done using the variational structure, the Lagrangian density being

$$
L(u, v)=\nabla u \nabla v-\frac{\lambda}{2} u^{2}-\frac{\mu}{2} v^{2}-\frac{1}{p+1} u^{p+1}-\frac{1}{q+1} v^{q+1},
$$

and is work in preparation [7]. We note that for the subcritical case, i.e. when $p$ and $q$ are below the critical hyperbola (1.2), the variational methods and results mirror those for the scalar equation when $1<p<\frac{n+2}{n-2}[8,9,15]$.

Ground states are also important for computing the optimal constants in Sobolev inequalities. For instance using (1.5) and (1.7) one can determine the best constant for the embeddings $\mathcal{D}^{1,2} \hookrightarrow L^{\frac{2 n}{n-2}}$ and $\mathcal{D}^{2,2} \hookrightarrow L^{\frac{2 n}{n-4}}$ respectively (see e.g. [10, 13, 14]). In general, the minimizer of (1.10) corresponds to the embedding $\mathcal{D}^{2, \frac{q+1}{q}} \hookrightarrow$ $L^{p+1}$.

\section{Existence AND UNIQUENESS}

Before proving uniqueness, let us first discuss the Lions result and the relation between (1.1) and (1.8). The proper function space on which to consider the functional (1.3) is

$$
(u, v) \in \mathcal{D}^{1, r} \times \mathcal{D}^{1, s}, \quad r=\frac{n(q+1)}{n q-q-1}, s=\frac{n(p+1)}{n p-p-1} .
$$

Critical points of (1.3) are also contained in $\mathcal{D}^{2, \frac{q+1}{q}} \times \mathcal{D}^{2, \frac{p+1}{p}}$ and are therefore not only weak but also classical solutions of (1.1). The corresponding critical values are 
given by

$$
\frac{2}{n} \int \nabla u \nabla v=\frac{2}{n} \int|u|^{p+1}=\frac{2}{n} \int|v|^{q+1}=\frac{2}{n} \int|\Delta v|^{\frac{p+1}{p}}=\frac{2}{n} \int|\Delta u|^{\frac{q+1}{q}} .
$$

From [11] it follows that (1.10) is attained by a function $u \in \mathcal{D}^{2, \frac{q+1}{q}}$ and that, after an innocent scaling which does not affect the minimizing property, such a minimizer is a positive, radially symmetric solution of (1.8), decreasing in $|x|$. Setting $v^{q}=-\Delta u$ we then obtain a critical point $(u, v)$ of $(1.3)$. If we now define $I_{p, q}$ by (1.9), it is easily seen from (2.2) and (1.10) that $I_{p, q}$ is in fact the smallest nonzero critical value of $J_{p, q}$.

For the uniqueness we argue as follows. Consider the radial system

$$
-v^{\prime \prime}-\frac{n-1}{r} v^{\prime}=u^{p}, \quad-u^{\prime \prime}-\frac{n-1}{r} u^{\prime}=v^{q},
$$

and suppose that we have two normalized positive solution pairs, say $\left(u_{1}, v_{1}\right)$ and $\left(u_{2}, v_{2}\right)$, with boundary conditions

$$
u^{\prime}(0)=v^{\prime}(0)=u(\infty)=v(\infty)=0,
$$

Thus $u_{1}(0)=u_{2}(0)=1$ and writing $v_{1}(0)=\sigma_{1}$ and $v_{2}(0)=\sigma_{2}$, we must have that $\sigma_{1}$ and $\sigma_{2}$ are different, so we may assume that $\sigma_{1}<\sigma_{2}$.

Now suppose that there exists a first point $\infty>r_{0}>0$ such that $v_{1}\left(r_{0}\right)=v_{2}\left(r_{0}\right)$. Clearly $v_{1}^{\prime}\left(r_{0}\right) \geq v_{2}^{\prime}\left(r_{0}\right)$. Moreover, for $r \leq r_{0}$,

$$
u_{1}^{\prime}(r)=-r^{1-n} \int_{0}^{r} s^{n-1} v_{1}(s)^{q} d s>-r^{1-n} \int_{0}^{r} s^{n-1} v_{2}(s)^{q} d s=u_{2}^{\prime}(r),
$$

which immediately gives that $u_{1}(r)>u_{2}(r)$ for $0<r \leq r_{0}$. A similar reasoning then yields that $v_{1}^{\prime}\left(r_{0}\right)<v_{2}^{\prime}\left(r_{0}\right)$, which is a contradiction. Thus $v_{1}(r)<v_{2}(r)$ for all $0 \leq r<\infty$, and consequently $u_{1}^{\prime}(r)>u_{2}^{\prime}(r)$ and $u_{1}(r)>u_{2}(r)$ for $0<r<\infty$. Integrating $u_{1}^{\prime}$ and $u_{2}^{\prime}$ over $[r, R)$ and letting $R$ tend to $\infty$, invoking (2.4), yields $u_{1}(r)<u_{2}(r)$, which again is a contradiction, completing the proof of uniqueness.

\section{THE QUADRATIC SYSTEM}

Our results follow from a transformation of the radial version of (1.1) (see (2.3)) into a 4-dimensional quadratic system. We set (compare $[4,5])$

$$
x=\frac{r u^{\prime}}{u}, \quad y=\frac{r v^{\prime}}{v}, \quad z=\frac{r^{2} v^{q}}{u}, \quad w=\frac{r^{2} u^{p}}{v}, \quad t=\log r .
$$

This leads to

$$
\begin{gathered}
x^{\prime}=x(2-n-x)-z ; \\
y^{\prime}=y(2-n-y)-w ; \\
z^{\prime}=z(2-x+q y) ; \\
w^{\prime}=w(2+p x-y) .
\end{gathered}
$$

Note that this transformation maps the family (1.11) into the same orbit. The intersection of the (invariant) set $\{z>0, w>0\}$ with the 2-dimensional unstable 
manifold $W^{u}$ of the origin, contains precisely those radial solutions of (1.1) which have both components bounded and positive at $r=0$. Thus we only consider orbits in $W^{u}$. Moreover, if we let

$$
\mathcal{A}=\{2-n \leq x, y<0, z>0, w>0\},
$$

integral curves may leave $\mathcal{A}$ through $x=2-n$ or $y=2-n$. If they do it is easy to see that they cannot enter $\mathcal{A}$ again. In fact the corresponding solution $(u, v)$ then has at least one of its components hitting zero in finite time. The ground state therefore corresponds to an orbit in $W^{u}$ which is completely contained in $\mathcal{A}$. It is not difficult to show directly for the quadratic system that such an orbit exists. Its uniqueness (see Theorem 1) is maybe less obvious in this setting.

A precise analysis of the (local structure of the) critical points of this system can be done and gives a (partial) classification of possible behaviours of $u(r)$ and $v(r)$ as $r \rightarrow 0$ or $r \rightarrow \infty$ for all values of $p$ and $q$. Here we restrict ourselves to the critical case (1.2) when this 4-dimensional system has a conserved quantity, namely

$$
z^{\frac{n}{2(q+1)}} w^{\frac{n}{2(p+1)}}\left(x y+\frac{n}{p+1} y+\frac{n}{q+1} x+\frac{w}{p+1}+\frac{z}{q+1}\right) .
$$

In particular the paraboloid $\mathcal{P}$ given by

$$
x y+\frac{n}{p+1} y+\frac{n}{q+1} x+\frac{w}{p+1}+\frac{z}{q+1}=0,
$$

is left invariant. Note that this 3 -dimensional manifold contains $W^{u}$, so we restrict our attention to the flow on $\mathcal{P}$. Thus we eleminate $w$ from the system and obtain that the $y$-equation reduces to

$$
y^{\prime}=y(2+(p+1) x-y)+(p(n-2)-2)\left(x+\frac{z}{n}\right) .
$$

For the reduced 3-dimensional system for $x, y$ and $z$ we consider the orbits in $W^{u}$ which are completely contained in the set $\mathcal{A}$. First we show that the $\omega$-limit set of any such orbit is a singleton. Thus we have to exclude chaotic or oscillatory behaviour, which is well known to occur for general 3-dimensional systems.

The analysis is based on the location of two parabolas in the $x, z$-plane. The first one, $P_{1}$, is simply the set where $x^{\prime}=0$, so

$$
P_{1}=\{x(2-n-x)-z=0\} .
$$

Referring to the $x$-axis as the horizontal axis, we have that $x^{\prime}<0$ above $P_{1}$ and $x^{\prime}>0$ below $P_{1}$. The second parabola is related to the possible maxima and minima of $z(t)$. Note that if $z^{\prime}=0$, then $z^{\prime \prime}=z\left(q y^{\prime}-x^{\prime}\right)$. Since $z>0$ (whence $2-x+q y=0)$ this may be evaluated to be

$$
z^{\prime \prime}=z \frac{2}{n(n+2 p+2)}\left(a z+b_{0}+b_{1} x+b_{2} x^{2}\right) \quad \text { if } z>0 \text { and } z^{\prime}=0,
$$

where

$$
\begin{gathered}
a=(n+2 p+2)(n+p+1), \quad b_{0}=-2 n^{2}(p+1), \\
b_{1}=4 n(n-1) p+n\left(n^{2}+2 n-4\right), \quad b_{2}=n\left(n+p^{2}+4 p+3\right) .
\end{gathered}
$$

Thus a second parabola, $P_{2}$, emerges,

$$
P_{2}=\left\{a z+b_{0}+b_{1} x+b_{2} x^{2}=0\right\} .
$$


Above $P_{2}$ only nondegenerate positive minima of $z(t)$ can occur and below $P_{2}$ only nondegenerate positive maxima (when $z>0$ ). Moreover, and this is the crux of the argument, between $x=2-n$ and $x=0, P_{2}$ lies entirely above $P_{1}$. Indeed the two parabolas intersect in the points

$$
(x, z)=(2,-2 n)
$$

and

$$
(x, z)=\left(-\frac{n^{2}}{p(n-2)-2}, \frac{n^{2}\left(p(n-2)^{2}+4-2 n-n^{2}\right)}{(p(n-2)-2)^{2}}\right),
$$

while $P_{2}$ intersects the positive $z$-axis. This implies that when $x^{\prime}(t)>0, z(t)$ cannot have a local positive minimum.

Now suppose that $x(t)$ has a minimum followed by a maximum. These two extrema lie on $P_{1}$, and in between, the solution lies below or possibly on $P_{1}$. We also have that when $x^{\prime}=0$, i.e. on $P_{1}$, then $x^{\prime \prime}=-z^{\prime}$. If the $x$-minimum is degenerate we have, since $P_{1}$ lies below $P_{2}$, that in this minimum $z(t)$ has a nondegenerate maximum. If it is not, then $z^{\prime}=-x^{\prime \prime}<0$. Likewise, if the $x$-maximum is degenerate, it coincides with a nondegenerate maximum for $z$ and if it is not, then $z^{\prime}=-x^{\prime \prime}>0$. In all four possible combinations, it follows that between the two $x$-extrema there must be a minimum of $z(t)$. This contradicts the fact that below $P_{2}$ the only extrema of $z(t)$ are nondegenerate maxima.

This argument shows that for $x(t)$ there are only two possibilities. Either it is decreasing all the way, or it first decreases to a minimum and then remains increasing. Let us now first discuss the latter case.

After the minimum of $x(t)$ the solution is below $P_{1}$, so from there on the only extrema of $z(t)$ are nondegenerate maxima. Consequently $z(t)$ is eventually monotone and thus as $t \rightarrow \infty$, both $x(t)$ and $z(t)$ converge to a finite limit below or on $P_{1}$. Using the $y$-equation and the fact that the orbit lies in the set $\mathcal{A}$, it follows that also $y(t)$ converges. We conclude that the $\omega$-limit set is a singleton.

On the other hand, if $x(t)$ is always decreasing, the orbit lies above $P_{1}$. Again we consider $z(t)$. If it oscillates as $t \rightarrow \infty$, its minima are above $P_{2}$ and its maxima below $P_{2}$. This gives a sequence of points $\left(x_{n}, z_{n}\right)(n=1,2, \ldots)$ where the odd indices correspond to maxima and the even indices correspond to minima of $z(t)$ (notice that the first extremum is a maximum in view of the fact that the orbit lies in the unstable manifold $W^{u}$ of the origin.) The sequence $x_{n}$ is decreasing so its limit $\xi$ exists. Let $(\xi, \zeta)$ be the corresponding point on $P_{2}$. We claim that $z_{n} \rightarrow \zeta$. Indeed for the maxima, which are below $P_{2}$, we must have $\lim \sup z_{2 k+1} \leq \zeta$, while for the minima, which are above $P_{2}$, the same reasoning gives $\lim \inf z_{2 k} \geq \zeta$. Hence $\lim z_{n}=\zeta$ and therefore also $\lim z(t)=\zeta$. As above this implies that the third coordinate $y(t)$ converges as well, so that the $\omega$-limit set is again a singleton.

Finally, if $z(t)$ does not oscillate, it is eventually monotone, either increasing or decreasing. Clearly it suffices now to exclude the possibility of $z(t)$ tending to infinity. But this would imply that $x^{\prime}(t) \rightarrow-\infty$, while $x(t) \geq 2-n$, impossible. It follows that both $z(t)$ and $y(t)$ converge.

Conclusion: any orbit in $W^{u}$ contained in $\mathcal{A}$ has an $\omega$-limit set which is a singleton (in $\mathcal{A}$ ). These can only be equilibria of the reduced system, and to know which, we have to examine these points and their local structure.

The reduced system has the following critical points $(x, y, z)$ :

$$
(0,0,0), \quad(0,2,0), \quad(2,0,-2 n),
$$




$$
\begin{aligned}
& \left(-\frac{n^{2}}{p(n-2)-2}, 2-n, \frac{n^{2}\left(p(n-2)^{2}+4-2 n-n^{2}\right)}{(p(n-2)-2)^{2}}\right), \\
& p_{1}=(2-n, 2-n, 0), \quad p_{2}=(2-n, 2-p(n-2), 0) .
\end{aligned}
$$

The point with the nonzero $z$-coordinate lies in the halfspace $\{z<0\}$ if $q>\frac{n}{n-2}$, which is certainly the case here because we assumed that $p \leq \frac{n+2}{n-2}$. Thus apart from the origin the only other points in $\mathcal{A}$ are $p_{1}$ and, provided $p \leq \frac{n}{n-2}$, the point $p_{2}$. The stable manifold of the origin is contained in $\{z=0\}$. Therefore every orbit in $W^{u}$ which stays in $\mathcal{A}$ forever is heteroclinic and goes into another critical point. If

$$
\frac{n}{n-2} \leq p \leq \frac{n+2}{n-2}
$$

there is only one candidate, namely $p_{1}=(2-n, 2-n, 0)$.

On the other hand, if

$$
\frac{2}{n-2}<p<\frac{n}{n-2}
$$

the limit is either $p_{1}$ or $p_{2}$. However in this case, $p_{1}$ has only a one-dimensional stable manifold which is contained in $\{y=2-n\}$ (in fact it is easily seen from the $w$-equation that it is also contained in $\{w=0\}$ ), so the limit can only be $p_{2}$. (In the next section we give the precise local structures near $p_{1}$ and $p_{2}$.)

The above analysis shows that the ground states have the following property.

$$
\begin{gathered}
\lim _{r \rightarrow \infty} \frac{r v^{\prime}(r)}{v(r)}=2-n \quad \text { if } \quad p \geq \frac{n}{n-2}, \\
\lim _{r \rightarrow \infty} \frac{r v^{\prime}(r)}{v(r)}=2-p(n-2) \quad \text { if } \quad p \leq \frac{n}{n-2},
\end{gathered}
$$

and

$$
\lim _{r \rightarrow \infty} \frac{r u^{\prime}(r)}{u(r)}=2-n .
$$

These limits are roughly speaking the exponents of $v$ and $u$ at infinity. In the next section we shall make this more precise.

\section{Local BeHAViour and the PROOF of Theorem 2}

It remains to improve the results at the end of the previous section so as to establish the asymptotics asserted in the introduction. This will follow from a local analysis around the critical points of the quadratic system.

4.1. The case $p>\frac{n}{n-2}$. We have seen in the previous section that the orbit containing the ground states goes into the critical point $p_{1}=(2-n, 2-n, 0)$ because it is the only candidate. The linearization of the reduced system around this point is given by the matrix

$$
\left[\begin{array}{ccc}
n-2 & 0 & -1 \\
-n & n-p(n-2) & \frac{p(n-2)-2}{n} \\
0 & 0 & n-q(n-2)
\end{array}\right] .
$$

Thus we have two negative eigenvalues, $\lambda_{1}=n-p(n-2)$ with eigenvector $(0,1,0)$ and $\lambda_{2}=n-q(n-2)$ with eigenvector $\left(p(n-2)-2,0, n^{2}\right)$, and one positive 
eigenvalue $\lambda_{3}=n-2$ with eigenvector $(2-p(n-2), n, 0)$. In particular it follows that the convergence is of exponential type. This implies that as $r=\exp (t) \rightarrow \infty$,

$$
\frac{r u^{\prime}(r)}{u(r)}=2-n+o\left(r^{-\lambda}\right) \text { with } \lambda>0
$$

It is a trivial exercise to show that consequently $r^{n-2} u(r)$ converges to a positive number. For $v(r)$ the argument is the same.

4.2. The case $p<\frac{n}{n-2}$. Now the critical point $p_{1}=(2-n, 2-n, 0)$ cannot be the limit because its stable manifold is only 1-dimensional and contained in $\{y=2-n\}$. The stable manifold is actually a straight line in the plane $\{y=2-n\}$, and is given by the equation $-n x+\frac{p(n-2)-2}{n} z=n(n-2)$. Thus the limit must be the critical point $p_{2}=(2-n, 2-p(n-2), 0)$ which enters the set $\mathcal{A}$ as $p$ crosses the Serrin exponent. The corresponding matrix is

$$
\left[\begin{array}{ccc}
n-2 & 0 & -1 \\
p(2-(n-2) p) & p(n-2)-n & \frac{p(n-2)-2}{n} \\
0 & 0 & -2(p+1))
\end{array}\right],
$$

which now has two negative eigenvalues and one positive eigenvalue. The rest of the argument is the same as in the previous subsection. The additional relation between $a$ and $b$ follows from the fact that the corresponding value of $w$ is now nonzero.

4.3. The case $p=\frac{n}{n-2}$. This case is more involved in view of a zero eigenvalue of the the critical point $(2-n, 2-n, 0)$ which now coincides with the point in the subsection above. If we set $y=2-n$ we obtain as a limit equation for $z$ that

$$
z^{\prime}=z\left(\frac{n^{2}}{2-n}-x\right)
$$

Combining with the $x$-equation we obtain a 2-dimensional limit system for $x$ and $z$. Linearizing this limit system around the critical point $(x, z)=(2-n, 0)$ we obtain the matrix

$$
\left[\begin{array}{cc}
n-2 & -1 \\
0 & -4 \frac{n-1}{n-2}
\end{array}\right]
$$

which has one positive and one negative eigenvalue, the latter with eigenvector $\left(n-2, n^{2}\right)$. It follows that the projection of the orbit containing the ground states on to the plane $\{y=2-n\}$ is asymptotically tangent to this eigenvector and that the convergence of $x(t)$ and $z(t)$ is again of exponential type. Thus the asymptotics of $u(r)$ are the same as before.

The heteroclinic orbit enters $p_{1}=p_{2}$ via a centermanifold (as in the nondegenerate case this orbit cannot enter $p_{1}$ via the stable manifold of $p_{1}$ ). From the theory of center manifolds (see e.g. [2], [3]) it then follows that the $x$ and $z$ variables converge exponentially, as indicated above, and the rate of convergence of $y$ as $t$ goes to $\infty$ is given by $y(t)+n-2=t^{-1}+O\left(t^{-2+\epsilon}\right)$. From the latter (1.15) now follows. 


\section{REFERENCES}

[1] Brezis, H. and L. Nirenberg, Positive solutions of nonlinear elliptic equations involving critical Sobolev exponents, Comm. Pure Appl. Math. 36 (1983), 437-477. MR 84h:35059

[2] Carr, J., Applications of centre manifold theory, Appl. Math. Sc. 35, Springer Verlag, 1981. MR 83g:34039

[3] Chow, S.N. and J.K. Hale, Methods of Bifurcation Theorem, Grundl. der math. Wiss 251, Springer Verlag, 1982. MR 84e:58019

[4] Hulshof, J., Similarity solutions of the porous medium equation with sign changes, J. Math. Anal. Appl., 157, 1991, 75-111. MR 92f:35082

[5] Hulshof, J., Similarity solutions of the $k-\epsilon$ model for turbulence, Report W93-11, Leiden University, 1993. (To appear as Similarity solutions of Barenblatt's model for turbulence, SIAM J. Math. Anal.)

[6] Hulshof, J., A local analysis of similarity solutions of the thin film equation, Report W94-22, Leiden University, 1994.

[7] Hulshof, J, E. Mitidieri and R.C.A.M. van der Vorst, Strongly indefinite systems with critical Sobolev exponents, Report W95-15, Leiden University, 1995.

[8] Hulshof, J. and R.C.A.M. van der Vorst, Positive solutions of the equation $\Delta u+u^{p}=0$ on a bounded domain, course notes, Report W93-06, Leiden University, 1993.

[9] Hulshof, J. and R.C.A.M. van der Vorst, Differential Systems with Strongly Indefinite Variational Structure, J. Funct. Anal., 114, 1993, 32-58. MR 94g:35073

[10] Lieb, E., Sharp constants in the Hardy-Littlewood-Sobolev and related inequalities, Ann. Math. 118 (1983), 349-374. MR 86j:42010

[11] Lions, P.L., The concentration-compactness principle in the calculus of variations, part 1, Rev. Mat. Iberoam. 1 (1985), 145-201. MR 87c:49007

[12] Mitidieri, E., A Rellich type identity and applications, Comm. PDE 18 (1993), 125-151. MR 94c:26016

[13] Van der Vorst, R.C.A.M., Best constant for the embedding of the space $H^{2} \cap H_{0}^{1}$ into $L^{2 N /(N-4)}$, Differential and Integral Equations 6 (1992), 259-276. MR 94b:46053

[14] Wang, X.J., Sharp constant in a Sobolev inequality, Nonlinear Anal. 20 (1993), 261-268. MR 94g:35035

[15] Felmer, P. and D. G. de Figueiredo, On super-quadratic elliptic systems, Trans. A. M. S. 343 (1994), 99-116.

Mathematical Department of the Leiden University, Niels Bohrweg 12333 CA LeiDEN, The NetherLands

E-mail address: hulshof@wi.leidenuniv.nl

WorldWideWeb: http://www.wi.leidenuniv.nl/home/hulshof/

Center for Dynamical Systems and Nonlinear Studies, Georgia Institute of TechnOlogy, Atlanta, Georgia 30308-0190

E-mail address: rvander@math.gatech.edu 